\title{
Buckling and free vibration analysis of laminated composite plates using an efficient and simple higher order shear deformation theory
}

\author{
Belkacem Adim $^{1,4}$, Tahar Hassaine Daouadji ${ }^{1,3, a}$, Boussad AbBes ${ }^{2}$ and A. Rabahi ${ }^{1,4}$ \\ 1 Département de génie civil, Université Ibn Khaldoun Tiaret, BP 78 Zaaroura, 14000 Tiaret, Algérie \\ 2 Laboratoire GRESPI, Campus du Moulin de la Housse, BP 1039, 51687 Reims Cedex 2, France \\ 3 Laboratoire de Géomatique et Développement Durable, Université Ibn Khaldoun, Tiaret, Algérie \\ ${ }^{4}$ Laboratoire de Technologie Industrielle, Université Ibn Khaldoun, Tiaret, Algérie
}

Received 13 February 2015, Accepted 21 November 2015

\begin{abstract}
In this paper, the buckling and free vibration analysis of laminated composite plates using an efficient and simple higher order shear deformation theory are examined by using a refined shear deformation theory. This theory is based on the assumption that the transverse displacements consist of bending and shear components where the bending components do not contribute to shear forces, and likewise, the shear components do not contribute to bending moments. The most interesting feature of this theory is that it allows for parabolic distributions of transverse shear stresses across the plate thickness and satisfies the conditions of zero shear stresses at the top and bottom surfaces of the plate without using shear correction factors. The number of independent unknowns in the present theory is four, as against five in other shear deformation theories. In this analysis, the equations of motion for simply supported thick laminated rectangular plates are derived and obtained through the use of Hamilton's principle. The closedform solutions of anti-symmetric cross-ply and angle- ply laminates are obtained using Navier solution. Numerical results of the present study are compared with three-dimensional elasticity solutions and results of the first-order and the other higher-order theories reported in the literature. It can be concluded that the proposed theory is accurate and simple in solving the buckling and free vibration behaviors of laminated composite plates.
\end{abstract}

Key words: Analytical solutions / laminated composite plates / higher-order shear deformation theory / buckling / free vibration / Navier solution

\section{Introduction}

Laminated composite plates are widely used in industry and new fields of technology. Due to the high degrees of anisotropy and the low rigidity in transverse shear of the plates, the Kirchhoff hypothesis as a classical theory is no longer adequate. The hypothesis states that the normal to the midplane of a plate remains straight and normal after deformation because of the negligible transverse shear effects. Refined theories without this assumption have been used recently. The classical laminate plate theory CLPT underpredicts deflections and over predicts frequencies as well as buckling loads with moderately thick plates. Many shear deformation theories accounting for transverse shear effects have been developed to overcome the deficiencies of the CLPT. The first-order

${ }^{a}$ Corresponding author: daouadjitah@yahoo.fr shear deformation theories FSDT based on Reissner [1] and Mindlin [2] account for the transverse shear effects by the way of linear variation of in-plane displacements through the thickness. A number of shear deformation theories have been proposed to date. The first such theory for laminated isotropic plates was apparently [3]. This theory was generalized to laminated anisotropic plates in reference [4]. It was shown in references [5-7], the FSDT violates equilibrium conditions at the top and bottom faces of the plate, shear correction factors are required to rectify the unrealistic variation of the shear strain/stress through the thickness. In order to overcome the limitations of FSDT, higher-order shear deformation theories HSDT, since which involve higher-order terms in Taylor's expansions of the displacements in the thickness coordinate, were developed by Librescu [8], Levinson [9], Bhimaraddi and Stevens [10], Reddy [11], Ren [12], Kant and Pandya [13], and Mohan et al. [14]. A good review 
of these theories for the analysis of laminated composite plates is available in references [15-19]. A refined plate theory using only two unknown functions was developed by Shimpi [20] for isotropic plates, and was extended by Shimpi and Patel $[21,22]$ for orthotropic plates. The most interesting feature of this theory is that it does not require shear correction factors, and has strong similarities with the classical plate theory in some aspects such as governing equation, boundary conditions and moment expressions.

In this paper, a refined and simple theory of plates is presented and applied to the investigation of buckling and free vibration behavior of laminated composite plates. This theory is based on the assumption that the in-plane and transverse displacements consist of bending and shear components where the bending components do not contribute to shear forces, and likewise, the shear components do not contribute to bending moments. The most interesting feature of this theory is that it allows for parabolic distributions of transverse shear stresses across the plate thickness and satisfies zero shear stress conditions at the top and bottom surfaces of the plate without using shear correction factors. The equations of motion are derived using Hamilton's principle. The fundamental frequencies are found by solving an eigenvalue equation. The results obtained by the present method are compared with solutions and results of the first-order and the other higher-order theories.

\section{Refined plate theory for laminated composite plates}

\subsection{Basic assumptions}

Consider a rectangular plate of total thickness h composed of $n$ orthotropic layers with the coordinate system as shown in Figure 1. Assumptions of the refined plate's theory are as follows:

- The displacements are small in comparison with the plate thickness and, therefore, strains involved are infinitesimal.

- The transverse displacement $w$ includes three components of bending $w_{\mathrm{b}}$ and shear $w_{\mathrm{s}}$. These components are functions of coordinates $x, y$, and time $t$ only.

$$
w(x, y, t)=w_{\mathrm{b}}(x, y, t)+w_{\mathrm{s}}(x, y, t)
$$

- The transverse normal stress $\sigma_{z}$ is negligible in comparison with in-plane stresses $\sigma_{x}$ and $\sigma_{y}$.

- The displacements $U$ in $x$-direction and $V$ in $y$ direction consist of extension, bending, and shear components:

$$
U=u+u_{\mathrm{b}}+u_{\mathrm{s}}, V=v+v_{\mathrm{b}}+v_{\mathrm{s}}
$$

- The bending components $u_{\mathrm{b}}$ and $v_{\mathrm{b}}$ are assumed to be similar to the displacements given by the classical plate theory. Therefore, the expression for $u_{\mathrm{b}}$ and $v_{\mathrm{b}}$ can be given as:

$$
u_{\mathrm{b}}=-z \frac{\partial w_{\mathrm{b}}}{\partial x}, \quad v_{\mathrm{b}}=-z \frac{\partial w_{\mathrm{b}}}{\partial y}
$$

- The shear components $u_{\mathrm{s}}$ and $v_{\mathrm{s}}$ give rise, in conjunction with $w_{\mathrm{s}}$, to the parabolic variations of shear strains $\gamma_{x z}, \gamma_{y z}$ and hence to shear stresses $\sigma_{x z}, \sigma_{y z}$ through the thickness of the plate in such a way that shear stresses $\sigma_{x z}, \sigma_{y z}$ are zero at the top and bottom faces of the plate. Consequently, the expression for $u_{\mathrm{s}}$ and $v_{\mathrm{s}}$ can be given as:

$$
u_{\mathrm{s}}=f(z) \frac{\partial w_{\mathrm{s}}}{\partial x}, \quad v_{\mathrm{s}}=f(z) \frac{\partial w_{\mathrm{s}}}{\partial y}
$$

\subsection{Kinematics}

Based on the assumptions made in the preceding section, the displacement field can be obtained using Equations (1)-(3) as:

$$
\begin{aligned}
u(x, y, z, t) & =u(x, y, t)-z \frac{\partial w_{\mathrm{b}}}{\partial x}+f(z) \frac{\partial w_{\mathrm{s}}}{\partial x} \\
v(x, y, z, t) & =v(x, y, t)-z \frac{\partial w_{\mathrm{b}}}{\partial y}+f(z) \frac{\partial w_{\mathrm{s}}}{\partial y} \\
w(x, y, z, t) & =w_{\mathrm{b}}(x, y, t)+w_{\mathrm{s}}(x, y, t)
\end{aligned}
$$

where $u$ and $v$ are the mid-plane displacements of the plate in the $x$ and $y$ direction, respectively; $w_{\mathrm{b}}$ and $w_{\mathrm{s}}$ are the bending and shear components of transverse displacement, respectively, while $f(z)$ represents shape functions determining the distribution of the transverse shear strains and stresses along the thickness and is given as the present model; the function $f(z)$ is an hyperbolic shape function (Hyperbolic Shear Deformation Theory):

$$
f(z)=z\left[1+\frac{3 \pi}{2} \operatorname{sech}^{2}\left(\frac{1}{2}\right)\right]-\frac{3 \pi}{2} h \tanh \left(\frac{z}{h}\right)
$$

It should be noted that unlike the first-order shear deformation theory, this theory does not require shear correction factors. The strains associated with the displacements in Equation (4) are:

$$
\begin{aligned}
\varepsilon_{x} & =\varepsilon_{x}^{0}+z k_{x}^{\mathrm{b}}+f k_{x}^{\mathrm{s}} \\
\varepsilon_{y} & =\varepsilon_{y}^{0}+z k_{y}^{\mathrm{b}}+f k_{y}^{\mathrm{s}} \\
\gamma_{x y} & =\gamma_{x y}^{0}+z k_{x y}^{\mathrm{b}}+f k_{x y}^{\mathrm{s}} \\
\gamma_{y z} & =g \gamma_{y z}^{\mathrm{s}} \\
\gamma_{x z} & =g \gamma_{x z}^{\mathrm{s}} \\
\varepsilon_{z} & =0
\end{aligned}
$$



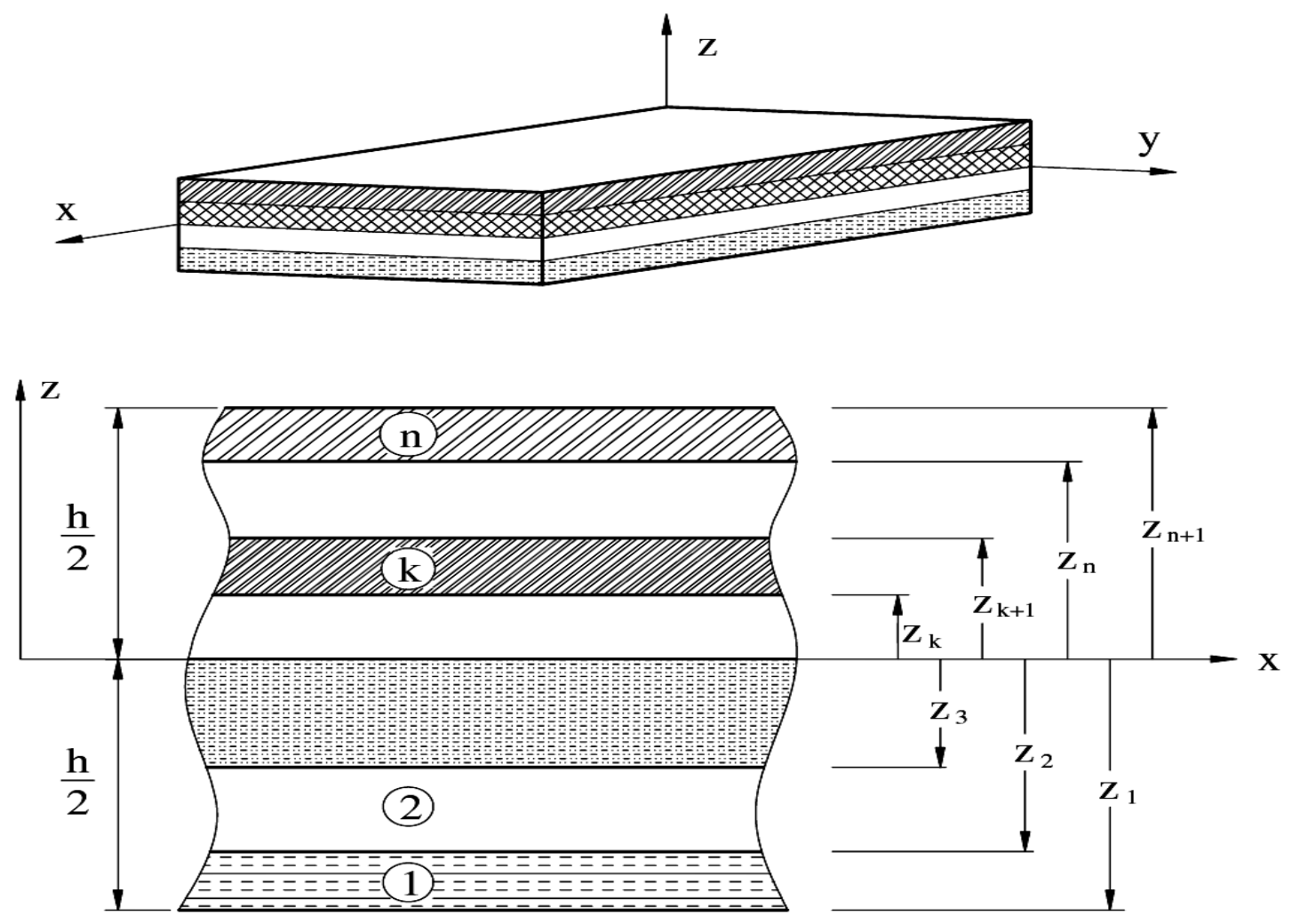

Fig. 1. Coordinate system and layer numbering used for a typical laminated plate.

where:

$$
\begin{aligned}
\varepsilon_{x}^{0} & =\frac{\partial u}{\partial x}, \quad k_{x}^{\mathrm{b}}=-\frac{\partial^{2} w_{\mathrm{b}}}{\partial x^{2}}, \quad k_{x}^{\mathrm{s}}=-\frac{\partial^{2} w_{\mathrm{s}}}{\partial x^{2}} \\
\varepsilon_{y}^{0} & =\frac{\partial v}{\partial y}, \quad k_{y}^{\mathrm{b}}=-\frac{\partial^{2} w_{\mathrm{b}}}{\partial y^{2}}, \quad k_{y}^{\mathrm{s}}=-\frac{\partial^{2} w_{\mathrm{s}}}{\partial y^{2}} \\
\gamma_{x y}^{0} & =\frac{\partial u}{\partial y}+\frac{\partial v}{\partial x}, \quad k_{x y}^{\mathrm{b}}=-2 \frac{\partial^{2} w_{\mathrm{b}}}{\partial x \partial y}, \\
k_{x y}^{\mathrm{s}} & =-2 \frac{\partial^{2} w_{\mathrm{s}}}{\partial x \partial y}, \quad \gamma_{y z}^{\mathrm{s}}=\frac{\partial w_{\mathrm{s}}}{\partial y}, \quad \gamma_{x z}^{\mathrm{s}}=\frac{\partial w_{\mathrm{s}}}{\partial x}
\end{aligned}
$$

$g(z)=1-f^{\prime}(z)$ and $f^{\prime}(z)=\frac{\mathrm{d} f(z)}{\mathrm{d} z}$.

\subsection{Constitutive equations}

Under the assumption that each layer possesses a plane of elastic symmetry parallel to the $x-y$ plane, the constitutive equations for a layer can be written as

$$
\left\{\begin{array}{l}
\sigma_{x} \\
\sigma_{y} \\
\sigma_{x y} \\
\sigma_{y z} \\
\sigma_{x z}
\end{array}\right\}=\left[\begin{array}{ccccc}
Q_{11} & Q_{12} & 0 & 0 & 0 \\
Q_{12} & Q_{22} & 0 & 0 & 0 \\
0 & 0 & Q_{66} & 0 & 0 \\
0 & 0 & 0 & Q_{44} & 0 \\
0 & 0 & 0 & 0 & Q_{55}
\end{array}\right]\left\{\begin{array}{c}
\varepsilon_{x} \\
\varepsilon_{y} \\
\gamma_{x y} \\
\gamma_{y z} \\
\gamma_{x z}
\end{array}\right\}
$$

where $Q_{i j}$ are the plane stress-reduced stiffnesses, and are known in terms of the engineering constants in the material axes of the layer:

$$
\begin{aligned}
& Q_{11}=\frac{E_{11}}{1-\nu_{12} \nu_{21}}, Q_{22}=\frac{E_{22}}{1-\nu_{12} \nu_{21}}, Q_{12}=\frac{\nu_{12} E_{22}}{1-\nu_{12} \nu_{21}} \\
& Q_{66}=G_{12}, \quad Q_{44}=G_{23}, \quad Q_{55}=G_{13}
\end{aligned}
$$

Since the laminate is made of several orthotropic layers with their material axes oriented arbitrarily with respect to the laminate coordinates, the constitutive equations of each layer must be transformed to the laminate coordinates $(x, y, z)$. The stress-strain relations in the laminate coordinates of the $k$ th layer are given as

$$
\left\{\begin{array}{c}
\sigma_{x} \\
\sigma_{y} \\
\sigma_{x y} \\
\sigma_{y z} \\
\sigma_{x z}
\end{array}\right\}=\left[\begin{array}{ccccc}
\bar{Q}_{11} & \bar{Q}_{12} & \bar{Q}_{16} & 0 & 0 \\
\bar{Q}_{12} & \bar{Q}_{22} & \bar{Q}_{26} & 0 & 0 \\
\bar{Q}_{16} & \bar{Q}_{26} & \bar{Q}_{66} & 0 & 0 \\
0 & 0 & 0 & \bar{Q}_{44} & \bar{Q}_{45} \\
0 & 0 & 0 & \bar{Q}_{45} & \bar{Q}_{55}
\end{array}\right]^{(k)}\left\{\begin{array}{c}
\varepsilon_{x} \\
\varepsilon_{y} \\
\gamma_{x y} \\
\gamma_{y z} \\
\gamma_{x z}
\end{array}\right\}
$$




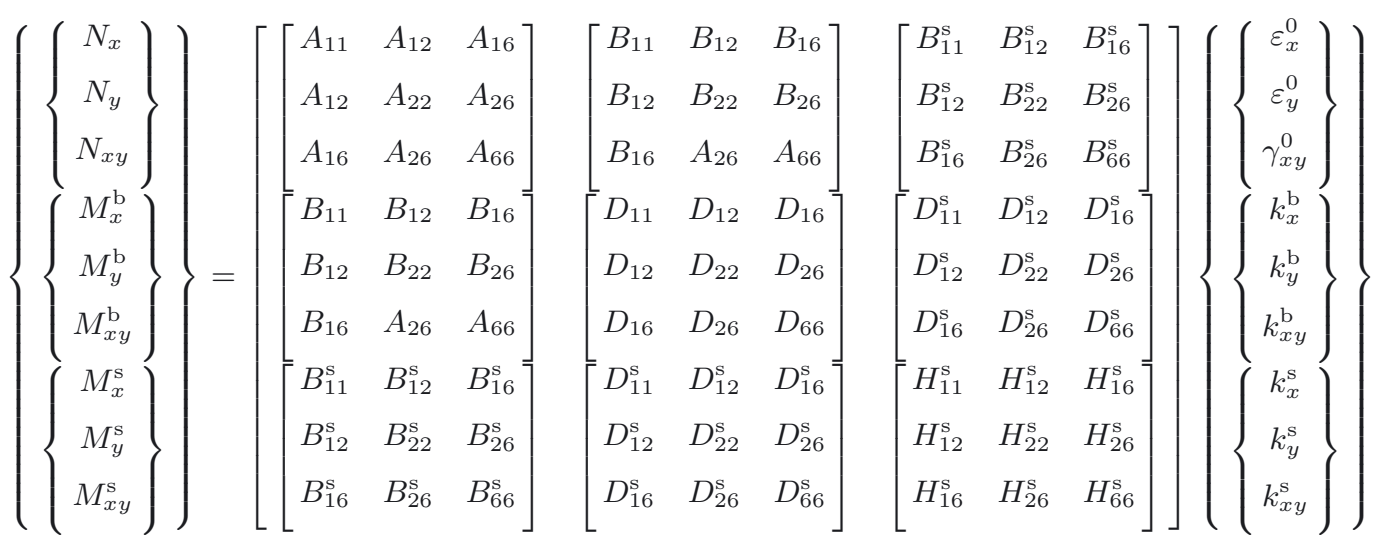

$$
\begin{aligned}
& \left\{\begin{array}{l}
Q_{y z}^{\mathrm{s}} \\
Q_{x z}^{\mathrm{s}}
\end{array}\right\}=\left[\begin{array}{cc}
A_{44}^{\mathrm{s}} & A_{45}^{\mathrm{s}} \\
A_{45}^{\mathrm{s}} & A_{55}^{\mathrm{s}}
\end{array}\right]\left\{\begin{array}{c}
\gamma_{y z}^{\mathrm{s}} \\
\gamma_{x z}^{\mathrm{s}}
\end{array}\right\}
\end{aligned}
$$

where $\bar{Q}_{i j}$ are the transformed material constants given as

$$
\begin{aligned}
\bar{Q}_{11}= & Q_{11} \cos ^{4} \theta+2\left(Q_{12}+2 Q_{66}\right) \sin ^{2} \theta \cos ^{2} \theta+Q_{22} \sin ^{4} \theta \\
\bar{Q}_{12}= & \left(Q_{11}+Q_{22}-4 Q_{66}\right) \sin ^{2} \theta \cos ^{2} \theta+Q_{12}\left(\sin ^{4} \theta+\cos ^{4} \theta\right) \\
\bar{Q}_{22}= & Q_{11} \sin ^{4} \theta+2\left(Q_{12}+2 Q_{66}\right) \sin ^{2} \theta \cos ^{2} \theta+Q_{22} \cos ^{4} \theta \\
\bar{Q}_{16}= & \left(Q_{11}-Q_{12}-2 Q_{66}\right) \sin \theta \cos ^{3} \theta \\
& +\left(Q_{12}-Q_{22}+2 Q_{66}\right) \sin ^{3} \theta \cos \theta \\
\bar{Q}_{26}= & \left(Q_{11}-Q_{12}-2 Q_{66}\right) \sin ^{3} \theta \cos \theta \\
& +\left(Q_{12}-Q_{22}+2 Q_{66}\right) \sin \theta \cos ^{3} \theta \\
\bar{Q}_{66}= & \left(Q_{11}+Q_{22}-2 Q_{12}-2 Q_{66}\right) \sin ^{2} \theta \cos ^{2} \theta \\
& +Q_{66}\left(\sin ^{4} \theta+\cos ^{4} \theta\right) \\
\bar{Q}_{44}= & Q_{44} \cos ^{2} \theta+Q_{55} \sin ^{2} \theta \\
\bar{Q}_{45}= & \left(Q_{55}-Q_{44}\right) \cos \theta \sin \theta \\
\bar{Q}_{55}= & Q_{55} \cos ^{2} \theta+Q_{44} \sin ^{2} \theta
\end{aligned}
$$

In which $\theta$ is the angle between the global $x$-axis and the local $x$-axis of each lamina.

\subsection{Governing equations}

The strain energy of the plate can be written as

$$
\begin{array}{rl}
U=\frac{1}{2} \int_{V} \sigma_{i j} \varepsilon_{i j} & \mathrm{~d} V=\frac{1}{2} \int_{V}\left(\sigma_{x} \varepsilon_{x}+\sigma_{y} \varepsilon_{y}\right. \\
& \left.+\sigma_{x y} \gamma_{x y}+\sigma_{y z} \gamma_{y z}+\sigma_{x z} \gamma_{x z}\right) \mathrm{d} V
\end{array}
$$

Substituting Equations (5) and (9) into Equation (11) and integrating through the thickness of the plate, the strain energy of the plate can be rewritten as

$$
\begin{aligned}
U & =\frac{1}{2} \int_{A}\left\{N_{x} \varepsilon_{x}^{0}+N_{y} \varepsilon_{y}^{0}+N_{x y} \gamma_{x y}^{0}+M_{x}^{\mathrm{b}} k_{x}^{\mathrm{b}}+M_{y}^{\mathrm{b}} k_{y}^{\mathrm{b}}\right. \\
& +M_{x y}^{\mathrm{b}} k_{x y}^{\mathrm{b}}+M_{x}^{\mathrm{s}} k_{x}^{\mathrm{s}}+M_{y}^{\mathrm{s}} k_{y}^{\mathrm{s}}+M_{x y}^{\mathrm{s}} k_{x y}^{\mathrm{s}}+Q_{y z}^{\mathrm{s}} \gamma_{y z}^{\mathrm{s}} \\
& \left.+Q_{x z}^{\mathrm{s}} \gamma_{x z}^{\mathrm{s}}\right\} \mathrm{d} x \mathrm{~d} y
\end{aligned}
$$

where the stress resultants $N, M$, and $Q$ are defined by

$$
\begin{aligned}
\left(N_{x}, N_{y}, N_{x y}\right) & =\int_{-h / 2}^{h / 2}\left(\sigma_{x}, \sigma_{y}, \sigma_{x y}\right) \mathrm{d} z \\
& =\sum_{k=1}^{N} \int_{z_{k}}^{z_{k+1}}\left(\sigma_{x}, \sigma_{y}, \sigma_{x y}\right) \mathrm{d} z \\
\left(M_{x}^{\mathrm{b}}, M_{y}^{\mathrm{b}}, M_{x y}^{\mathrm{b}}\right) & =\int_{-h / 2}^{h / 2}\left(\sigma_{x}, \sigma_{y}, \sigma_{x y}\right) z \mathrm{~d} z \\
& =\sum_{k=1}^{N} \int_{z_{k}}^{z_{k+1}}\left(\sigma_{x}, \sigma_{y}, \sigma_{x y}\right) z \mathrm{~d} z \\
\left(M_{x}^{\mathrm{s}}, M_{y}^{\mathrm{s}}, M_{x y}^{\mathrm{s}}\right) & =\int_{-h / 2}^{h / 2}\left(\sigma_{x}, \sigma_{y}, \sigma_{x y}\right) f \mathrm{~d} z \\
& =\sum_{k=1}^{N} \int_{z_{k}}^{z_{k+1}}\left(\sigma_{x}, \sigma_{y}, \sigma_{x y}\right) f \mathrm{~d} z \\
\left(Q_{x z}^{\mathrm{s}}, Q_{y z}^{\mathrm{s}}\right) & =\int_{-h / 2}^{h / 2}\left(\sigma_{x z}, \sigma_{y z}\right) g \mathrm{~d} z \\
& =\sum_{k=1}^{N} \int_{z_{k}}^{z_{k+1}}\left(\sigma_{x z}, \sigma_{y z}\right) g \mathrm{~d} z
\end{aligned}
$$

Substituting Equation (9) into Equation (13) and integrating through the thickness of the plate, the stress resultants are given as:

See equations (14a) and (14b) above 
where $A_{i j}, B_{i j}$, etc., are the plate stiffnesses, defined by

$$
\begin{aligned}
& \left(A_{i j}, B_{i j}, D_{i j}, B_{i j}^{\mathrm{s}}, D_{i j}^{\mathrm{s}}, H_{i j}^{\mathrm{s}}\right) \\
& h / 2 \\
& =\int_{-h / 2} \bar{Q}_{i j}\left(1, z, z^{2}, f(z), z f(z), f^{2}(z)\right) \mathrm{d} z,(i, j)=(1,2,6)
\end{aligned}
$$$$
A_{i j}^{\mathrm{s}}=\int_{-h / 2}^{h / 2} \bar{Q}_{i j}[g(z)]^{2} \mathrm{~d} z,(i, j)=(4,5)
$$

The work done by applied forces can be written as:

$$
\begin{array}{r}
V=\frac{1}{2} \int_{A}\left[N_{x}^{0} \frac{\partial^{2}\left(w_{\mathrm{b}}+w_{\mathrm{s}}\right)}{\partial x^{2}}+N_{y}^{0} \frac{\partial^{2}\left(w_{\mathrm{b}}+w_{\mathrm{s}}\right)}{\partial y^{2}}\right. \\
\left.+2 N_{x y}^{0} \frac{\partial^{2}\left(w_{\mathrm{b}}+w_{\mathrm{s}}\right)}{\partial x \partial y}\right] \mathrm{d} x \mathrm{~d} y
\end{array}
$$

where $N_{x}^{0}, N_{y}^{0}$ and $N_{x y}^{0}$ are in-plane distributed forces.

The kinetic energy of the plate can be written as

$$
\begin{aligned}
T= & \frac{1}{2} \int_{V} \rho \ddot{u}_{i i} \mathrm{~d} V=\frac{1}{2} \int_{A}\left\{\delta u\left(I_{1} \ddot{u}-I_{2} \frac{\partial \ddot{w}_{\mathrm{b}}}{\partial x} w-I_{4} \frac{\partial \ddot{w}_{\mathrm{s}}}{\partial x}\right)\right. \\
& +\delta v\left(I_{1} \ddot{v}-I_{2} \frac{\partial \ddot{w}_{\mathrm{b}}}{\partial y}-I_{4} \frac{\partial \ddot{w}_{\mathrm{s}}}{\partial y}\right) \\
& +\delta w_{\mathrm{b}}\left[I_{1}\left(\ddot{w}_{\mathrm{b}}+\ddot{w}_{\mathrm{s}}\right)+I_{2}\left(\frac{\partial \ddot{u}}{\partial x}+\frac{\partial \ddot{v}}{\partial y}\right)\right. \\
& \left.-I_{3}\left(\frac{\partial^{2} \ddot{w}_{\mathrm{b}}}{\partial x^{2}}+\frac{\partial^{2} \ddot{w}_{\mathrm{b}}}{\partial y^{2}}\right)-I_{5}\left(\frac{\partial^{2} \ddot{w}_{\mathrm{s}}}{\partial x^{2}}+\frac{\partial^{2} \ddot{w}_{\mathrm{s}}}{\partial y^{2}}\right)\right] \\
& +\delta w_{\mathrm{s}}\left[I_{1}\left(\ddot{w}_{\mathrm{b}}+\ddot{w}_{\mathrm{s}}\right)+I_{4}\left(\frac{\partial \ddot{u}}{\partial x}+\frac{\partial \ddot{v}}{\partial y}\right)\right. \\
& \left.\left.-I_{5}\left(\frac{\partial^{2} \ddot{w}_{\mathrm{b}}}{\partial x^{2}}+\frac{\partial^{2} \ddot{w}_{\mathrm{b}}}{\partial y^{2}}\right)-I_{6}\left(\frac{\partial^{2} \ddot{w}_{\mathrm{s}}}{\partial x^{2}}+\frac{\partial^{2} \ddot{w}_{\mathrm{s}}}{\partial y^{2}}\right)\right]\right\} \mathrm{d} x \mathrm{~d} y
\end{aligned}
$$

where $\rho$ is the mass of density of the plate and $I_{i}$ are the inertias defined by

$$
\left(I_{1}, I_{2}, I_{3}, I_{4}, I_{5}, I_{6}\right)=\int_{-h / 2}^{h / 2} \rho\left(1, z, z^{2}, f(z), z f(z),[f(z)]^{2}\right) \mathrm{d} z
$$

Hamilton's principle [18] is used herein to derive the equations of motion appropriate to the displacement field and the constitutive equation. The principle can be stated in analytical form as

$$
\int_{0}^{t} \delta(U+V-T) \mathrm{d} t=0
$$

where $\delta$ indicates a variation with respect to $x$ and $y$.

Substituting Equations (12), (16) and (17) into Equation (19) and integrating the equation by parts, collecting the coefficients of $\delta u, \delta v, \delta w_{\mathrm{b}}$ and $\delta w_{\mathrm{s}}$, the equations of motion for the laminate plate are obtained as follows:

$$
\begin{aligned}
\delta u & : \frac{\partial N_{x}}{\partial x}+\frac{\partial N_{x y}}{\partial y}=I_{1} \ddot{u}-I_{2} \frac{\partial \ddot{w}_{\mathrm{b}}}{\partial x}-I_{4} \frac{\partial \ddot{w}_{\mathrm{s}}}{\partial x} \\
\delta v & : \frac{\partial N_{x y}}{\partial x}+\frac{\partial N_{y}}{\partial y}=I_{1} \ddot{v}-I_{2} \frac{\partial \ddot{w}_{\mathrm{b}}}{\partial y}-I_{4} \frac{\partial \ddot{w}_{\mathrm{s}}}{\partial y} \\
\delta w_{\mathrm{b}} & : \frac{\partial^{2} M_{x}^{\mathrm{b}}}{\partial x^{2}}+2 \frac{\partial^{2} M_{x y}^{\mathrm{b}}}{\partial x \partial y}+\frac{\partial^{2} M_{y}^{\mathrm{b}}}{\partial y^{2}}+N(w) \\
& =I_{1}\left(\ddot{w}_{\mathrm{b}}+\ddot{w}_{\mathrm{s}}\right)+I_{2}\left(\frac{\partial \ddot{u}}{\partial x}+\frac{\partial \ddot{v}}{\partial y}\right) \\
& -I_{3}\left(\frac{\partial^{2} \ddot{w}_{\mathrm{b}}}{\partial x^{2}}+\frac{\partial^{2} \ddot{w}_{\mathrm{b}}}{\partial y^{2}}\right)-I_{5}\left(\frac{\partial^{2} \ddot{w}_{\mathrm{s}}}{\partial x^{2}}+\frac{\partial^{2} \ddot{w}_{\mathrm{s}}}{\partial y^{2}}\right) \\
\delta w_{\mathrm{s}} & : \frac{\partial^{2} M_{x}^{\mathrm{s}}}{\partial x^{2}}+2 \frac{\partial^{2} M_{x y}^{\mathrm{s}}}{\partial x \partial y}+\frac{\partial^{2} M_{y}^{\mathrm{s}}}{\partial y^{2}}+\frac{\partial Q_{x z}^{\mathrm{s}}}{\partial x}+\frac{\partial Q_{y z}^{\mathrm{s}}}{\partial y}+N(w) \\
& =I_{1}\left(\ddot{w}_{\mathrm{b}}+\ddot{w}_{\mathrm{s}}\right)+I_{4}\left(\frac{\partial \ddot{u}}{\partial x}+\frac{\partial \ddot{v}}{\partial y}\right) \\
& -I_{5}\left(\frac{\partial^{2} \ddot{w}_{\mathrm{b}}}{\partial x^{2}}+\frac{\partial^{2} \ddot{w}_{\mathrm{b}}}{\partial y^{2}}\right)-I_{6}\left(\frac{\partial^{2} \ddot{w}_{\mathrm{s}}}{\partial x^{2}}+\frac{\partial^{2} \ddot{w}_{\mathrm{s}}}{\partial y^{2}}\right) \quad(20)
\end{aligned}
$$

where $N(w)$ is defined by

$$
\begin{aligned}
N(w)=N_{x}^{0} \frac{\partial^{2}\left(w_{\mathrm{b}}+w_{\mathrm{s}}\right)}{\partial x^{2}}+ & N_{y}^{0} \frac{\partial^{2}\left(w_{\mathrm{b}}+w_{\mathrm{s}}\right)}{\partial y^{2}} \\
& +2 N_{x y}^{0} \frac{\partial^{2}\left(w_{\mathrm{b}}+w_{\mathrm{s}}\right)}{\partial x \partial y}
\end{aligned}
$$

Equation (20) can be expressed in terms of displacements $\left(u, v, w_{\mathrm{b}}, w_{\mathrm{s}}\right)$ by substituting for the stress resultants from Equation (14). For homogeneous laminates, the equations of motion (20) take the form

See equations (22a)-(22d) next page.

\section{Analytical solutions}

\subsection{Analytical solutions for antisymmetric cross-ply laminates}

The Navier solutions can be developed for rectangular laminates with two sets of simply supported boundary conditions. For antisymmetric cross-ply laminates, the following plate stiffnesses are identically zero:

$$
\begin{aligned}
A_{16} & =A_{26}=D_{16}=D_{26}=D_{16}^{\mathrm{s}}=D_{26}^{\mathrm{s}}=H_{16}^{\mathrm{s}}=H_{26}^{\mathrm{s}}=0 \\
B_{12} & =B_{26}=B_{16}=B_{66}=B_{12}^{\mathrm{s}}=B_{16}^{\mathrm{s}}=B_{26}^{\mathrm{s}}=B_{66}^{\mathrm{s}} \\
& =A_{45}^{\mathrm{s}}=0 \\
B_{22} & =-B_{11}, B_{22}^{\mathrm{s}}=-B_{11}^{\mathrm{s}}
\end{aligned}
$$




$$
\begin{aligned}
& A_{11} \frac{\partial^{2} u}{\partial x^{2}}+2 A_{16} \frac{\partial^{2} u}{\partial x \partial y}+A_{66} \frac{\partial^{2} u}{\partial y^{2}}+A_{16} \frac{\partial^{2} v}{\partial x^{2}}+\left(A_{12}+A_{66}\right) \frac{\partial^{2} v}{\partial x \partial y}+A_{26} \frac{\partial^{2} v}{\partial y^{2}} \\
& -B_{11} \frac{\partial^{3} w_{\mathrm{b}}}{\partial x^{3}}-3 B_{16} \frac{\partial^{3} w_{\mathrm{b}}}{\partial x^{2} \partial y}-\left(B_{12}+2 B_{66}\right) \frac{\partial^{3} w_{\mathrm{b}}}{\partial x \partial y^{2}}-B_{26} \frac{\partial^{3} w_{\mathrm{b}}}{\partial y^{3}} \\
& -B_{11}^{\mathrm{s}} \frac{\partial^{3} w_{\mathrm{s}}}{\partial x^{3}}-3 B_{16}^{\mathrm{s}} \frac{\partial^{3} w_{\mathrm{s}}}{\partial x^{2} \partial y}-\left(B_{12}^{\mathrm{s}}+2 B_{66}^{\mathrm{s}}\right) \frac{\partial^{3} w_{\mathrm{s}}}{\partial x \partial y^{2}}-B_{26}^{\mathrm{s}} \frac{\partial^{3} w_{\mathrm{s}}}{\partial y^{3}}=I_{1} \ddot{u}-I_{2} \frac{\partial \ddot{w}_{\mathrm{b}}}{\partial x}-I_{4} \frac{\partial \ddot{w}_{\mathrm{s}}}{\partial x} \\
& A_{16} \frac{\partial^{2} u}{\partial x^{2}}+\left(A_{12}+A_{66}\right) \frac{\partial^{2} u}{\partial x \partial y}+A_{26} \frac{\partial^{2} u}{\partial y^{2}}+A_{66} \frac{\partial^{2} v}{\partial x^{2}}+2 A_{26} \frac{\partial^{2} v}{\partial x \partial y}+A_{22} \frac{\partial^{2} v}{\partial y^{2}} \\
& -B_{16} \frac{\partial^{3} w_{\mathrm{b}}}{\partial x^{3}}-\left(B_{12}+2 B_{66}\right) \frac{\partial^{3} w_{\mathrm{b}}}{\partial x^{2} \partial y}-3 B_{26} \frac{\partial^{3} w_{\mathrm{b}}}{\partial x \partial y^{2}}-B_{22} \frac{\partial^{3} w_{\mathrm{b}}}{\partial y^{3}} \\
& -B_{16}^{\mathrm{s}} \frac{\partial^{3} w_{\mathrm{s}}}{\partial x^{3}}-\left(B_{12}^{\mathrm{s}}+2 B_{66}^{\mathrm{s}}\right) \frac{\partial^{3} w_{\mathrm{s}}}{\partial x^{2} \partial y}-3 B_{26}^{\mathrm{s}} \frac{\partial^{3} w_{\mathrm{s}}}{\partial x \partial y^{2}}-B_{22}^{\mathrm{s}} \frac{\partial^{3} w_{\mathrm{s}}}{\partial y^{3}}=I_{1} \ddot{v}_{0}-I_{2} \frac{\partial \ddot{w}_{\mathrm{b}}}{\partial y}-I_{4} \frac{\partial \ddot{w}_{\mathrm{s}}}{\partial y} \\
& B_{11} \frac{\partial^{3} u}{\partial x^{3}}+3 B_{16} \frac{\partial^{3} u}{\partial x^{2} \partial y}+\left(B_{12}+2 B_{66}\right) \frac{\partial^{3} u}{\partial x \partial y^{2}}+B_{26} \frac{\partial^{3} u}{\partial y^{3}} \\
& +B_{16} \frac{\partial^{3} v}{\partial x^{3}}+\left(B_{12}+2 B_{66}\right) \frac{\partial^{3} v}{\partial x^{2} \partial y}+3 B_{26} \frac{\partial^{3} v}{\partial x \partial y^{2}}+B_{22} \frac{\partial^{3} v}{\partial y^{3}} \\
& -D_{11} \frac{\partial^{4} w_{\mathrm{b}}}{\partial x^{4}}-4 D_{16} \frac{\partial^{4} w_{\mathrm{b}}}{\partial x^{3} \partial y}-2\left(D_{12}+2 D_{66}\right) \frac{\partial^{4} w_{\mathrm{b}}}{\partial x^{2} \partial y^{2}}-4 D_{26} \frac{\partial^{4} w_{\mathrm{b}}}{\partial x \partial y^{3}}-D_{22} \frac{\partial^{4} w_{\mathrm{b}}}{\partial y^{4}} \\
& -D_{11}^{\mathrm{s}} \frac{\partial^{4} w_{\mathrm{s}}}{\partial x^{4}}-4 D_{16}^{\mathrm{s}} \frac{\partial^{4} w_{\mathrm{s}}}{\partial x^{3} \partial y}-2\left(D_{12}^{\mathrm{s}}+2 D_{66}^{\mathrm{s}}\right) \frac{\partial^{4} w_{\mathrm{s}}}{\partial x^{2} \partial y^{2}}-4 D_{26}^{\mathrm{s}} \frac{\partial^{4} w_{\mathrm{s}}}{\partial x \partial y^{3}}-D_{22}^{\mathrm{s}} \frac{\partial^{4} w_{\mathrm{s}}}{\partial y^{4}}+N(w) \\
& =I_{1}\left(\ddot{w}_{\mathrm{b}}+\ddot{w}_{\mathrm{s}}\right)+I_{2}\left(\frac{\partial \ddot{u}}{\partial x}+\frac{\partial \ddot{v}}{\partial y}\right)-I_{3}\left(\frac{\partial^{2} \ddot{w}_{\mathrm{b}}}{\partial x^{2}}+\frac{\partial^{2} \ddot{w}_{\mathrm{b}}}{\partial y^{2}}\right)-I_{5}\left(\frac{\partial^{2} \ddot{w}_{\mathrm{s}}}{\partial x^{2}}+\frac{\partial^{2} \ddot{w}_{\mathrm{s}}}{\partial y^{2}}\right) \\
& B_{11}^{\mathrm{s}} \frac{\partial^{3} u}{\partial x^{3}}+3 B_{16}^{\mathrm{s}} \frac{\partial^{3} u}{\partial x^{2} \partial y}+\left(B_{12}^{\mathrm{s}}+2 B_{66}^{\mathrm{s}}\right) \frac{\partial^{3} u}{\partial x \partial y^{2}}+B_{26}^{\mathrm{s}} \frac{\partial^{3} u}{\partial y^{3}} \\
& +B_{16}^{\mathrm{s}} \frac{\partial^{3} v}{\partial x^{3}}+\left(B_{12}^{\mathrm{s}}+2 B_{66}^{\mathrm{s}}\right) \frac{\partial^{3} v}{\partial x^{2} \partial y}+3 B_{26}^{\mathrm{s}} \frac{\partial^{3} v}{\partial x \partial y^{2}}+B_{22}^{\mathrm{s}} \frac{\partial^{3} v}{\partial y^{3}} \\
& -D_{11}^{\mathrm{s}} \frac{\partial^{4} w_{\mathrm{b}}}{\partial x^{4}}-4 D_{16}^{\mathrm{s}} \frac{\partial^{4} w_{\mathrm{b}}}{\partial x^{3} \partial y}-2\left(D_{12}^{\mathrm{s}}+2 D_{66}^{\mathrm{s}}\right) \frac{\partial^{4} w_{\mathrm{b}}}{\partial x^{2} \partial y^{2}}-4 D_{26}^{\mathrm{s}} \frac{\partial^{4} w_{\mathrm{b}}}{\partial x \partial y^{3}}-D_{22}^{\mathrm{s}} \frac{\partial^{4} w_{\mathrm{b}}}{\partial y^{4}} \\
& -H_{11}^{\mathrm{s}} \frac{\partial^{4} w_{\mathrm{s}}}{\partial x^{4}}-4 H_{16}^{\mathrm{s}} \frac{\partial^{4} w_{\mathrm{s}}}{\partial x^{3} \partial y}-2\left(H_{12}^{\mathrm{s}}+2 H_{66}^{\mathrm{s}}\right) \frac{\partial^{4} w_{\mathrm{s}}}{\partial x^{2} \partial y^{2}}-4 H_{26}^{\mathrm{s}} \frac{\partial^{4} w_{\mathrm{s}}}{\partial x \partial y^{3}}-H_{22}^{\mathrm{s}} \frac{\partial^{4} w_{\mathrm{s}}}{\partial y^{4}} \\
& +A_{55}^{\mathrm{s}} \frac{\partial^{2} w_{\mathrm{s}}}{\partial x^{2}}+A_{44}^{\mathrm{s}} \frac{\partial^{2} w_{\mathrm{s}}}{\partial y^{2}}+2 A_{45}^{\mathrm{s}} \frac{\partial^{2} w_{\mathrm{s}}}{\partial x \partial y}+N(w) \\
& =I_{1}\left(\ddot{w}_{\mathrm{b}}+\ddot{w}_{\mathrm{s}}\right)+I_{4}\left(\frac{\partial \ddot{u}}{\partial x}+\frac{\partial \ddot{v}}{\partial y}\right)-I_{5}\left(\frac{\partial^{2} \ddot{w}_{\mathrm{b}}}{\partial x^{2}}+\frac{\partial^{2} \ddot{w}_{\mathrm{b}}}{\partial y^{2}}\right)-I_{6}\left(\frac{\partial^{2} \ddot{w}_{\mathrm{s}}}{\partial x^{2}}+\frac{\partial^{2} \ddot{w}_{\mathrm{s}}}{\partial y^{2}}\right)
\end{aligned}
$$


The following boundary conditions for antisymmetric cross-ply laminates can be written as

$$
\begin{aligned}
v(0, y) & =w_{\mathrm{b}}(0, y)=w_{\mathrm{s}}(0, y)=\frac{\partial w_{\mathrm{b}}}{\partial y}(0, y) \\
& =\frac{\partial w_{\mathrm{s}}}{\partial y}(0, y)=0 \\
v(a, y) & =w_{\mathrm{b}}(a, y)=w_{\mathrm{s}}(a, y)=\frac{\partial w_{\mathrm{b}}}{\partial y}(a, y) \\
& =\frac{\partial w_{\mathrm{s}}}{\partial y}(a, y)=0 \\
N_{x}(0, y) & =M_{x}^{\mathrm{b}}(0, y)=M_{x}^{\mathrm{s}}(0, y)=N_{x}(a, y)=M_{x}^{\mathrm{b}}(a, y) \\
& =M_{x}^{\mathrm{s}}(a, y)=0 \\
u(x, 0) & =w_{\mathrm{b}}(x, 0)=w_{\mathrm{s}}(x, 0)=\frac{\partial w_{\mathrm{b}}}{\partial x}(x, 0) \\
& =\frac{\partial w_{\mathrm{s}}}{\partial x}(x, 0)=0 \\
N_{y}(x, 0) & =M_{y}^{\mathrm{b}}(x, 0)=M_{y}^{\mathrm{s}}(x, 0)=N_{y}(x, b)=M_{y}^{\mathrm{b}}(x, b) \\
& =M_{y}^{\mathrm{s}}(x, b)=0 \\
u(x, b) & =w_{\mathrm{b}}(x, b)=w_{\mathrm{s}}(x, b)=\frac{\partial w_{\mathrm{b}}}{\partial x}(x, b) \\
& \frac{\partial w_{\mathrm{s}}}{\partial x}(x, b)=0 \\
&
\end{aligned}
$$

The boundary conditions in Equation (24) are satisfied by the following expansions

$$
\begin{gathered}
u=\sum_{m=1}^{\infty} \sum_{n=1}^{\infty} U_{m n} \mathrm{e}^{i \omega t} \cos (\alpha x) \sin (\beta y) \\
v=\sum_{m=1}^{\infty} \sum_{n=1}^{\infty} V_{m n} \mathrm{e}^{i \omega t} \sin (\alpha x) \cos (\beta y) \\
w_{\mathrm{b}}=\sum_{m=1}^{\infty} \sum_{n=1}^{\infty} W_{b m n} \mathrm{e}^{i \omega t} \sin (\alpha x) \sin (\beta y) \\
w_{\mathrm{s}}=\sum_{m=1}^{\infty} \sum_{n=1}^{\infty} W_{s m n} \mathrm{e}^{i \omega t} \sin (\alpha x) \sin (\beta y)
\end{gathered}
$$

where $U_{m n}, V_{m n}, W_{b m n}$ and $W_{s m n}$ unknown parameters must be determined, $\omega$ is the eigen frequency associated with $(m, n)$ the eigen-mode, and $\alpha=\frac{m \pi}{a}$ and $\beta=\frac{n \pi}{b}$.

Substituting Equations (23) and (25) into Equation (22), the Navier solution of antisymmetric cross-ply laminates can be determined from equations

$$
\begin{aligned}
& {\left[\begin{array}{cccc}
s_{11} & s_{12} & s_{13} & s_{14} \\
s_{12} & s_{22} & s_{23} & s_{24} \\
s_{13} & s_{23} & s_{33}+k & s_{34}+k \\
s_{14} & s_{24} & s_{34}+k & s_{44}+k
\end{array}\right]\left\{\begin{array}{c}
U_{m n} \\
V_{m n} \\
W_{b m n} \\
W_{s m n}
\end{array}\right\}} \\
& +\left[\begin{array}{cccc}
m_{11} & 0 & 0 & 0 \\
0 & m_{22} & 0 & 0 \\
0 & 0 & m_{33} & m_{34} \\
0 & 0 & m_{34} & m s_{44}
\end{array}\right]\left\{\begin{array}{l}
\ddot{U}_{m n} \\
\ddot{V}_{m n} \\
\ddot{W}_{b m n} \\
\ddot{W}_{s m n}
\end{array}\right\}=\left\{\begin{array}{l}
0 \\
0 \\
0 \\
0
\end{array}\right\}
\end{aligned}
$$

where

$$
\begin{aligned}
s_{11} & =A_{11} \alpha^{2}+A_{66} \beta^{2}, s_{12}=\alpha \beta\left(A_{12}+A_{66}\right), \\
s_{13} & =-B_{11} \alpha^{3}, s_{14}=-B_{11}^{\mathrm{s}} \alpha^{3} \\
s_{22} & =A_{66} \alpha^{2}+A_{22} \beta^{2}, s_{23}=B_{11} \beta^{3}, s_{24}=B_{11}^{\mathrm{s}} \beta^{3} \\
s_{33} & =D_{11} \alpha^{4}+2\left(D_{12}+2 D_{66}\right) \alpha^{2} \beta^{2}+D_{22} \beta^{4} \\
s_{34} & =D_{11}^{\mathrm{s}} \alpha^{4}+2\left(D_{12}^{\mathrm{s}}+2 D_{66}^{\mathrm{s}}\right) \alpha^{2} \beta^{2}+D_{22}^{\mathrm{s}} \beta^{4} \\
s_{44} & =H_{11}^{\mathrm{s}} \alpha^{4}+2\left(H_{12}^{\mathrm{s}}+2 H_{66}^{\mathrm{s}}\right) \alpha^{2} \beta^{2}+H_{22}^{\mathrm{s}} \beta^{4} \\
& +A_{55}^{\mathrm{s}} \alpha^{2}+A_{44}^{\mathrm{s}} \beta^{2} \\
m_{11} & =m_{22}=I_{1}, m_{33}=I_{1}+I_{3}\left(\alpha^{2}+\beta^{2}\right) \\
m_{34} & =I_{1}+I_{5}\left(\alpha^{2}+\beta^{2}\right), m_{44}=I_{1}+I_{6}\left(\alpha^{2}+\beta^{2}\right), \\
k & =N_{x}^{0} \alpha^{2}+N_{y}^{0} \beta^{2}
\end{aligned}
$$

\subsection{Analytical solutions for antisymmetric angle-ply laminates}

For antisymmetric angle-ply laminates, the following plate stiffnesses are identically zero:

$$
\begin{aligned}
A_{16} & =A_{26}=D_{16}=D_{26}=D_{16}^{\mathrm{s}}=D_{26}^{\mathrm{s}}=H_{16}^{\mathrm{s}} \\
& =H_{26}^{\mathrm{s}}=0 \\
B_{11} & =B_{12}=B_{22}=B_{66}=B_{11}^{\mathrm{s}}=B_{12}^{\mathrm{s}}=B_{22}^{\mathrm{s}} \\
& =B_{66}^{\mathrm{s}}=A_{45}^{\mathrm{s}}=0
\end{aligned}
$$


The following boundary conditions for antisymmetric angle-ply laminates can be written as

$$
\begin{aligned}
u(0, y) & =w_{\mathrm{b}}(0, y)=w_{\mathrm{s}}(0, y)=\frac{\partial w_{\mathrm{b}}}{\partial y}(0, y) \\
& =\frac{\partial w_{\mathrm{s}}}{\partial y}(0, y)=0 \\
u(a, y) & =w_{\mathrm{b}}(a, y)=w_{\mathrm{s}}(a, y)=\frac{\partial w_{\mathrm{b}}}{\partial y}(a, y) \\
& =\frac{\partial w_{\mathrm{s}}}{\partial y}(a, y)=0 \\
N_{x y}(0, y) & =M_{x}^{\mathrm{b}}(0, y)=M_{x}^{\mathrm{s}}(0, y)=N_{x y}(a, y)=M_{x}^{\mathrm{b}}(a, y) \\
& =M_{x}^{\mathrm{s}}(a, y)=0 \\
v(x, 0) & =w_{\mathrm{b}}(x, 0)=w_{\mathrm{s}}(x, 0)=\frac{\partial w_{\mathrm{b}}}{\partial x}(x, 0) \\
& =\frac{\partial w_{\mathrm{s}}}{\partial x}(x, 0)=0 \\
v(x, b) & =w_{\mathrm{b}}(x, b)=w_{\mathrm{s}}(x, b)=\frac{\partial w_{\mathrm{b}}}{\partial x}(x, b) \\
& =\frac{\partial w_{\mathrm{s}}}{\partial x}(x, b)=0 \\
N_{x y}(x, 0) & =M_{y}^{\mathrm{b}}(x, 0)=M_{y}^{\mathrm{s}}(x, 0)=N_{x y}(x, b)=M_{y}^{\mathrm{b}}(x, b) \\
& =M_{y}^{\mathrm{s}}(x, b)=0
\end{aligned}
$$

The boundary conditions in Equation (29) are satisfied by the following expansions

$$
\begin{aligned}
u & =\sum_{m=1}^{\infty} \sum_{n=1}^{\infty} U_{m n} \mathrm{e}^{i \omega t} \sin (\alpha x) \cos (\beta y) \\
v & =\sum_{m=1}^{\infty} \sum_{n=1}^{\infty} V_{m n} \mathrm{e}^{i \omega t} \cos (\alpha x) \sin (\beta y) \\
w_{\mathrm{b}} & =\sum_{m=1}^{\infty} \sum_{n=1}^{\infty} W_{\mathrm{bmn}} \mathrm{e}^{i \omega t} \sin (\alpha x) \sin (\beta y) \\
w_{\mathrm{s}} & =\sum_{m=1}^{\infty} \sum_{n=1}^{\infty} W_{\mathrm{smn}} \mathrm{e}^{i \omega t} \sin (\alpha x) \sin (\beta y)
\end{aligned}
$$

Substituting Equations (28) and (30) into Equation (22), the equations of the form in Equation (26) are obtained with the following coefficients

$$
\begin{aligned}
s_{11}= & A_{11} \alpha^{2}+A_{66} \beta^{2}, s_{12}=\alpha \beta\left(A_{12}+A_{66}\right), \\
s_{13}= & -\left(3 B_{16} \alpha^{2} \beta+B_{26} \beta^{3}\right), s_{14}=-\left(3 B_{16}^{\mathrm{s}} \alpha^{2} \beta+B_{26}^{\mathrm{s}} \beta^{3}\right), \\
s_{22}= & A_{66} \alpha^{2}+A_{22} \beta^{2}, s_{23}=-\left(B_{16} \alpha^{3}+3 B_{26} \alpha \beta^{2}\right) \\
s_{24}= & -\left(B_{16}^{\mathrm{s}} \alpha^{3}+3 B_{26}^{\mathrm{s}} \alpha \beta^{2}\right), \\
s_{33}= & D_{11} \alpha^{4}+2\left(D_{12}+2 D_{66}\right) \alpha^{2} \beta^{2}+D_{22} \beta^{4}, \\
s_{34}= & D_{11}^{\mathrm{s}} \alpha^{4}+2\left(D_{12}^{\mathrm{s}}+2 D_{66}^{\mathrm{s}}\right) \alpha^{2} \beta^{2}+D_{22}^{\mathrm{s}} \beta^{4} \\
s_{44}= & H_{11}^{\mathrm{s}} \alpha^{4}+2\left(H_{12}^{\mathrm{s}}+2 H_{66}^{\mathrm{s}}\right) \alpha^{2} \beta^{2}+H_{22}^{\mathrm{s}} \beta^{4} \\
& +A_{55}^{\mathrm{s}} \alpha^{2}+A_{44}^{\mathrm{s}} \beta^{2}, \\
m_{11}= & m_{22}=I_{1}, m_{33}=I_{1}+I_{3}\left(\alpha^{2}+\beta^{2}\right), \\
m_{34}= & I_{1}+I_{5}\left(\alpha^{2}+\beta^{2}\right), m_{44}=I_{1}+I_{6}\left(\alpha^{2}+\beta^{2}\right), \\
k= & N_{x}^{0} \alpha^{2}+N_{y}^{0} \beta^{2}
\end{aligned}
$$

\section{Numerical results}

In this study, a buckling and free vibration analysis of anti-symmetrically cross-ply and angle-ply laminates composite plates by using the present shear deformation theory for laminated plates is suggested. The Navier solutions for free vibrations of laminated composite plates are found by solving eigen value equations. For the verification purpose, the results obtained by the present model are compared with those of the CLPT, FSDT, HSDT, and exact solution of three-dimensional elasticity. In all examples, a shear correction factor of $5 / 6$ is used for FSDT. The lamina properties shown in Table 1 are used. For convenience, the following nondimensionalizations are used in presenting the numerical results in graphical and tabular forms:

$$
\bar{N}=N_{c r}\left(\frac{a^{2}}{E_{2} h^{3}}\right), \quad \bar{\omega}=\omega \frac{a^{2}}{h} \sqrt{\frac{\rho}{E_{2}}}
$$

\subsection{Numerical results for buckling analysis}

For buckling analysis, the applied loads are assumed to be in-plane forces

$$
N_{x}^{0}=-N_{0}, N_{y}^{0}=\gamma N_{0}, \gamma=\frac{N_{x}^{0}}{N_{y}^{0}}, N_{x y}^{0}=0
$$

The buckling solution can be obtained from Equation (26) by setting the time derivative terms and transverse forces to zero:

$$
\begin{array}{r}
{\left[\begin{array}{cccc}
s_{11} & s_{12} & s_{13} & s_{14} \\
s_{12} & s_{22} & s_{23} & s_{24} \\
s_{13} & s_{23} & s_{33}-N_{0}\left(\alpha^{2}+\gamma \beta^{2}\right) & s_{34}-N_{0}\left(\alpha^{2}+\gamma \beta^{2}\right) \\
s_{14} & s_{24} & s_{34}-N_{0}\left(\alpha^{2}+\gamma \beta^{2}\right) & s_{44}-N_{0}\left(\alpha^{2}+\gamma \beta^{2}\right)
\end{array}\right]} \\
\times\left\{\begin{array}{c}
U_{m n} \\
V_{m n} \\
W_{b m n} \\
W_{s m n}
\end{array}\right\}=\left\{\begin{array}{l}
0 \\
0 \\
0 \\
0
\end{array}\right\} \quad(34)
\end{array}
$$

Following the procedure of condensation of variables to eliminate the in-plane displacements $U_{m n}$ and $V_{m n}$, the following system is obtained:

$$
\begin{aligned}
& {\left[\begin{array}{ll}
\bar{s}_{33}-N_{0}\left(\alpha^{2}+\gamma \beta^{2}\right) & \bar{s}_{34}-N_{0}\left(\alpha^{2}+\gamma \beta^{2}\right) \\
\bar{s}_{43}-N_{0}\left(\alpha^{2}+\gamma \beta^{2}\right) & \bar{s}_{44}-N_{0}\left(\alpha^{2}+\gamma \beta^{2}\right)
\end{array}\right]} \\
& \times\left\{\begin{array}{l}
W_{b m n} \\
W_{s m n}
\end{array}\right\}=\left\{\begin{array}{l}
0 \\
0
\end{array}\right\}
\end{aligned}
$$

where:

$$
\begin{aligned}
\bar{s}_{33} & =s_{33}-s_{13} \frac{b_{1}}{b_{0}}-s_{23} \frac{b_{2}}{b_{0}}, \bar{s}_{34}=s_{34}-s_{14} \frac{b_{1}}{b_{0}}-s_{24} \frac{b_{2}}{b_{0}}, \\
\bar{s}_{43} & =s_{34}-s_{13} \frac{b_{3}}{b_{0}}-s_{23} \frac{b_{4}}{b_{0}}, \bar{s}_{44}=s_{44}-s_{14} \frac{b_{3}}{b_{0}}-s_{24} \frac{b_{4}}{b_{0}} \\
b_{0} & =s_{11} s_{22}-s_{12}^{2}, b_{1}=s_{13} s_{22}-s_{12} s_{23}, b_{2}=s_{11} s_{23}-s_{12} s_{13}, \\
b_{3} & =s_{14} s_{22}-s_{12} s_{24}, b_{4}=s_{11} s_{24}-s_{12} s_{14}
\end{aligned}
$$


Table 1. The orthotropic material properties.

\begin{tabular}{cc}
\hline Material 1 [23] & $E_{1}=40 E_{2}, \mathrm{G}_{12}=\mathrm{G}_{13}=0.6 E_{2}, \mathrm{G}_{23}=0.5 E_{2}, \nu_{12}=0.25$ \\
\hline Material 2 $[25]$ & $E_{1}=40 E_{2}, \mathrm{G}_{12}=\mathrm{G}_{13}=0.5 E_{2}, \mathrm{G}_{23}=0.6 E_{2}, \nu_{12}=0.25$ \\
\hline Material 3 $[32]$ & $E_{1}=25 E_{2}, \mathrm{G}_{12}=\mathrm{G}_{13}=0.5 E_{2}, \mathrm{G}_{23}=0.2 E_{2}, \nu_{12}=0.25$ \\
\hline
\end{tabular}

Table 2. Nondimensional uniaxial buckling load of simply supported anti-symmetric cross-ply $(0 / 90 / \ldots)$ square laminates $(a / h=10)$.

\begin{tabular}{ccc}
\hline Number of layers & Theory & $\bar{N}$ \\
\hline & Present Model & 22.5821 \\
4 & Exact [23] & 21.2796 \\
& Reddy [11] & 22.5790 \\
& FSDT [24] & 22.8060 \\
CLPT & 30.3591 \\
\hline \multirow{4}{*}{6} & Present Model & 24.4605 \\
& Exact [23] & 23.6689 \\
& Reddy [11] & 24.4596 \\
& FSDT [24] & 24.5777 \\
\cline { 2 - 3 } 10 & CLPT & 33.5817 \\
\hline & Present Model & 25.4223 \\
& Exact [23] & 24.9636 \\
& Reddy [11] & 25.4225 \\
& FSDT [24] & 25.4500 \\
& CLPT & 35.2316 \\
\hline
\end{tabular}

For nontrivial solution, the determinant of the coefficient matrix in Equation (35) must be zero. This gives the following expression for buckling load:

$$
N_{0}=\left(\frac{1}{\alpha^{2}+\gamma \beta^{2}}\right)\left(\frac{\bar{s}_{33} \bar{s}_{44}-\bar{s}_{34} \bar{s}_{43}}{\bar{s}_{33}+\bar{s}_{44}-\bar{s}_{34}-\bar{s}_{43}}\right)
$$

A simply supported anti-symmetric cross-ply $(0 / 90)_{n}$ ( $n=2,3,5)$ square laminate subjected to uniaxial compressive load is considered. Table 2 shows a comparison between the results obtained using the various models and the three-dimensional elasticity solutions given by Noor [23]. The results clearly indicate that the present model gives more accurate results in predicting the buckling loads when compared to Reddy [11], and indicates that Reddy's theory is closer to the present model. Compared to the three-dimensional elasticity solution, the buckling loads predicted by present model, Reddy [11], and FSDT [24] are $6 \%$ to $7 \%$, respectively, for four-layer antisymmetric cross-ply $(0 / 90 / 0 / 90)$ square laminates. The effect of side-to-thickness ratio on buckling load of simply supported four-layer (0/90/0/90) square laminates is also presented in Figures 2 and 3 .

In Table 3, a simply supported two-layer antisymmetric angle-ply $(\theta /-\theta)$ square laminate subjected to uniaxial compressive loading is considered for the numerical values of nondimensional buckling load. The results are compared with higher order theory values reported by Ren [25]. For all values of side-to-thickness ratio and fiber orientation, the buckling loads predicted by the present
Table 3. Nondimensional uniaxial buckling load of simply supported two-layer $(\theta /-\theta)$ square laminates

\begin{tabular}{cccc}
\hline \multirow{2}{*}{$a / h$} & \multirow{2}{*}{ Theory } & \multicolumn{2}{c}{$\bar{N}$} \\
\cline { 3 - 4 } & & $\theta=30^{\circ}$ & $\theta=45^{\circ}$ \\
\hline \multirow{4}{*}{4} & Present Model & 9.5554 & 9.9145 \\
& Ren [25] & 9.5368 & 9.8200 \\
& Reddy [11] & 9.3391 & 8.2377 \\
& FSDT [24] & 7.5450 & 6.7858 \\
\hline \multirow{4}{*}{10} & Present Model & 17.2737 & 18.1473 \\
& Ren [25] & 15.7517 & 16.4558 \\
& Reddy [11] & 17.1269 & 18.1544 \\
& FSDT [24] & 16.6132 & 17.5522 \\
\hline \multirow{4}{*}{100} & Present Model & 20.5040 & 21.6662 \\
& Ren [25] & 20.4793 & 21.6384 \\
& Reddy [11] & 20.5017 & 21.6663 \\
& FSDT [24] & 20.4944 & 21.6576 \\
\hline
\end{tabular}

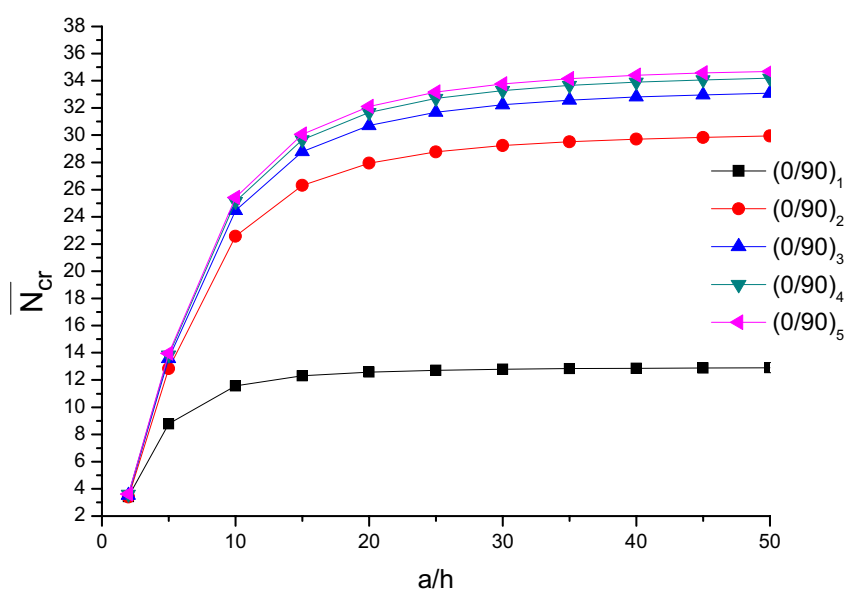

Fig. 2. The effect of side-to-thickness ratio on nondimensionalized uniaxial buckling load of simply supported antisymmetric cross-ply $(0 / 90)_{n}$ square laminates.

model and Reddy [11] are almost identical. For $a / h$ ratio equal to 4 and the fiber orientation equal to $30^{\circ}$, the buckling load values predicted by FSDT [24], Reddy [11], and present model are $18 \%$ to $2 \%$ lower as compared to the values obtained by Ren [25]. The results computed using all the five models are in a good agreement with those reported by Ren [25] for thin plates $(a / h=100)$. Figure 4 shows the effect of modulus ratio on nondimensionalized uniaxial buckling load of simply supported twolayer $(45 /-45)$ square laminate $\left(G_{12}=G_{13}=0.6 E_{2}\right.$, $\left.G_{23}=0.5 E_{2}, \nu_{12}=0.25, a / h=10\right)$. 


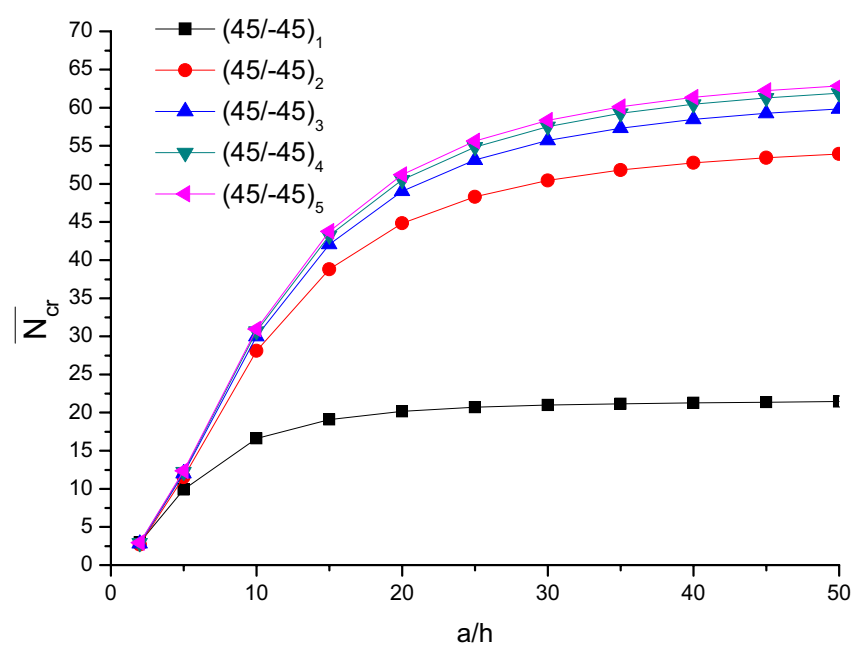

Fig. 3. The effect of side-to-thickness ratio on nondimensionalized uniaxial buckling load of simply supported antisymmetric angle-ply $(45 /-45)_{n}$ square laminates.

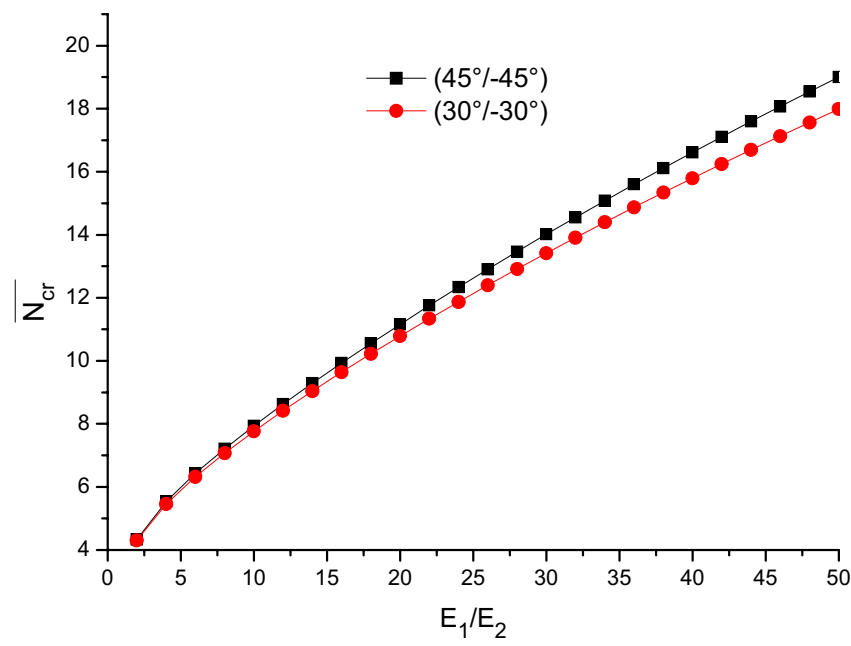

Fig. 4. The effect of modulus ratio on nondimensionalized deflection of simply supported two-layer anti-symmetric angleply square laminates under sinusoidal transverse load $(a / h=$ 10).

\subsection{Numerical results for free vibration analysis}

In the case of free vibration, the natural frequencies of the laminates can be obtained by setting the determinant of the coefficient of the following matrix to zero.

$$
\begin{gathered}
\left(\left[\begin{array}{llll}
s_{11} & s_{12} & s_{13} & s_{14} \\
s_{12} & s_{22} & s_{23} & s_{24} \\
s_{13} & s_{23} & s_{33} & s_{34} \\
s_{14} & s_{24} & s_{34} & s_{44}
\end{array}\right]-\omega^{2}\left[\begin{array}{cccc}
m_{11} & 0 & 0 & 0 \\
0 & m_{22} & 0 & 0 \\
0 & 0 & m_{33} & m_{34} \\
0 & 0 & m_{34} & m_{44}
\end{array}\right]\right) \\
\times\left\{\begin{array}{c}
U_{m n} \\
V_{m n} \\
W_{\mathrm{bmn}} \\
W_{\mathrm{smn}}
\end{array}\right\}=\left\{\begin{array}{l}
0 \\
0 \\
0 \\
0
\end{array}\right\}
\end{gathered}
$$

In Tables 4 and 5, the nondimensional fundamental frequencies of anti-symmetrically laminated cross-ply plates obtained by using different shear deformation theories are shown for various values of $a / h$ and modules ratios. It can be seen that, in general, the present model gives more accurate results in predicting the natural frequencies than those of Reddy [11], Karama [28] and the threedimensional elasticity solution given in reference [26]. It should be noted that unknown functions in present model are four; while the unknown functions in the FSDT [27] and higher-order shear deformation theories [11,28] are five. It can be concluded that the present model is not only accurate, but also simple in predicting the natural frequencies of laminated plates.

The variation of natural frequencies with respect to side-to-thickness ratio $a / h$ is presented in Tables 6 and 7 . The natural frequencies obtained using the present model is compared with Reddy's theory PSDT [11], Swaminathan [29] and FSDT [30]. In the case of thick plates $(a / h$ ratios $2,4,5$ and 10) there is a considerable difference between the results computed using the present and the theory's $[11,29,30]$. The variation of natural frequencies with respect to side-to-thickness ratio $a / h$ for different $E_{1} / E_{2}$ ratios is presented in Table 7 . For a four layered thick plate with $a / h$ ratio equal to 2 and $E_{1} / E_{2}$ ratio equal to 3 and 10 , the percentage differences in values predicted by present theory are $0.15 \%$ and $3.50 \%$ lower as compared to Reddy's theory PSDT [11] and Swaminathan [29]. At higher range of $E_{1} / E_{2}$ ratio equal to $20-40$, the percentage difference in values between both the theories is very much higher and Reddy's theory very much overpredicts the natural frequency values. For a four layered thick plate with $a / h$ ratio equal to 2 and $E_{1} / E_{2}$ ratio equal to 20 , 30 and 40, the percentage differences in values predicted by present theory are $6 \%, 8 \%$ and $9.50 \%$ lower as compared to the theory's $[11,29,30]$. The difference between the models tends to reduce for thin and relatively thin plates. Irrespective of the number of layers the percentage difference in values between the two theories $[11,29]$ increases with the increase in the degree of anisotropy. As the number of layers increases, the percentage difference in values between the two theories decreases significantly.

Dimensionless fundamental frequencies are given in Table 8 for various values of modulus ratio and ply number. The obtained results are compared with the exact 3D solutions reported by Reddy's theory [11]. Here also the results obtained by the present FSDT are almost identical with those predicted by existing FSDT [30]. This statement is also firmly demonstrated in Figures 5 and 6 in which the results obtained by the present theory are in excellent agreement for a wide range of thickness ratio $a / h$. According to Tables 7 and 8 the present results are in good agreement with the results of Reddy PSDT [11], Swaminathan [29] and Song Xiang [31].

\section{Conclusion}

A refined shear deformation theory of plates has been successfully developed for the buckling and free vibration of simply supported laminated plates. The theory allows for a square-law variation in the transverse shear strains 
B. Adim et al.: Mechanics \& Industry 17, 512 (2016)

Table 4. Nondimensional fundamental frequencies of anti-symmetric square plates at various values of orthotropy ratio with $a / h=5$.

\begin{tabular}{ccccccc}
\hline \multirow{2}{*}{ No. of layers } & Theory & \multicolumn{5}{c}{$E_{1} / E_{2}$} \\
\cline { 3 - 6 } & Exact [26] & 6.2578 & 6.9845 & 7.6745 & 8.1763 & 8.5625 \\
& Present Model & 6.2168 & 6.9881 & 7.8198 & 8.5028 & 9.0841 \\
$\left(0^{\circ} / 90^{\circ}\right)_{1}$ & Reddy [11] & 6.2169 & 6.9887 & 7.8210 & 8.5050 & 9.0871 \\
& Karama [28] & 6.2224 & 7.0066 & 7.8584 & 8.5630 & 9.1661 \\
& FSDT [27] & 6.2085 & 6.9392 & 7.7060 & 8.3211 & 8.8333 \\
\hline & Exact [26] & 6.5455 & 8.1445 & 9.4055 & 10.1650 & 10.6790 \\
& Present Model & 6.5009 & 8.1958 & 9.6273 & 10.5359 & 11.1728 \\
$\left(0^{\circ} / 90^{\circ}\right)_{2}$ & Reddy [11] & 6.5008 & 8.1954 & 9.6265 & 10.5348 & 11.1716 \\
& Karama [28] & 6.5034 & 8.1939 & 9.6201 & 10.5261 & 11.1628 \\
& FSDT [27] & 6.5043 & 8.2246 & 9.6885 & 10.6198 & 11.2708 \\
\hline & Exact [26] & 6.6100 & 8.4143 & 9.8398 & 10.6950 & 11.2720 \\
& Present Model & 6.5558 & 8.4053 & 9.9182 & 10.8546 & 11.5009 \\
$\left(0^{\circ} / 90^{\circ}\right)_{3}$ & Reddy [11] & 6.5558 & 8.4052 & 9.9181 & 10.8547 & 11.5012 \\
& Karama [28] & 6.5595 & 6.5595 & 9.9313 & 10.8756 & 11.5314 \\
& FSDT [27] & 6.5569 & 8.4183 & 9.9427 & 10.8828 & 11.5264 \\
\hline & Exact [26] & 6.6458 & 8.5625 & 10.0843 & 11.0027 & 11.6245 \\
& Present Model & 6.5842 & 8.5126 & 10.0671 & 11.0191 & 11.6721 \\
& Reddy [11] & 6.5842 & 8.5126 & 10.0674 & 11.0197 & 11.6730 \\
& Karama [28] & 6.5885 & 8.5229 & 10.0881 & 11.0522 & 11.7180 \\
& FSDT [27] & 6.5837 & 8.5132 & 10.0638 & 11.0058 & 11.6444 \\
\hline
\end{tabular}

Table 5. Nondimensional fundamental frequencies of anti-symmetric square plates at various values of $a / h$ with $E_{1} / E_{2}=40$.

\begin{tabular}{cccccccc}
\hline \multirow{2}{*}{ No. of layers } & Theory & \multicolumn{7}{c}{$a / h$} \\
\cline { 3 - 7 } & & 2 & 4 & 10 & 20 & 50 & 100 \\
\hline & Present Model & 5.7100 & 8.3507 & 10.5669 & 11.1048 & 11.2750 & 11.3001 \\
$\left(0^{\circ} / 90^{\circ}\right)_{1}$ & Karama [28] & 5.8948 & 8.4561 & 10.5964 & 11.1132 & 11.2764 & 11.3005 \\
& Reddy [11] & 5.7170 & 8.3546 & 10.5680 & 11.1052 & 11.2751 & 11.3002 \\
& FSDT [27] & 5.2104 & 8.0349 & 10.4731 & 11.0779 & 11.2705 & 11.2990 \\
& CLPT & 8.6067 & 10.4244 & 11.1537 & 11.2693 & 11.3023 & 11.3070 \\
\hline & Present Model & 5.7528 & 9.7366 & 14.8474 & 16.5737 & 17.1850 & 17.2784 \\
$\left(0^{\circ} / 90^{\circ}\right)_{2}$ & Karama [28] & 5.8129 & 9.7362 & 14.8338 & 16.5683 & 17.1840 & 17.2781 \\
& Reddy [11] & 5.7546 & 9.7357 & 14.8463 & 16.5733 & 17.1849 & 17.2784 \\
& FSDT [27] & 5.6656 & 9.8148 & 14.9214 & 16.6008 & 17.1899 & 17.2796 \\
& CLPT & 14.1036 & 16.3395 & 17.1448 & 17.2682 & 17.3032 & 17.3083 \\
\hline & Present Model & 5.8702 & 9.9870 & 15.4635 & 17.3774 & 18.0644 & 18.1699 \\
& Karama [28] & 5.9888 & 10.0323 & 15.4702 & 17.3787 & 18.0646 & 18.1699 \\
$\left(0^{\circ} / 90^{\circ}\right)_{3}$ & Reddy [11] & 5.8741 & 9.9878 & 15.4632 & 17.3772 & 18.0644 & 18.1698 \\
& FSDT [27] & 5.6992 & 9.9852 & 15.5010 & 17.3926 & 18.0673 & 18.1706 \\
& CLPT & 15.0895 & 17.2676 & 18.0461 & 18.1652 & 18.1990 & 18.2038 \\
\hline & Present Model & 5.9476 & 10.1226 & 15.7700 & 17.7743 & 18.4984 & 18.6097 \\
& Karama [28] & 6.0889 & 10.1854 & 15.7847 & 17.7784 & 18.4991 & 18.6099 \\
& Reddy [11] & 5.9524 & 10.1241 & 15.7700 & 17.7743 & 18.4984 & 18.6097 \\
& FSDT [27] & 5.7140 & 10.0628 & 15.7790 & 17.7800 & 18.4995 & 18.6100 \\
& CLPT & 15.6064 & 17.7314 & 18.4916 & 18.6080 & 18.6410 & 18.6457 \\
\hline
\end{tabular}


Table 6. Non-dimensionalized fundamental frequencies for a simply supported anti-symmetric angle-ply square laminated plate.

\begin{tabular}{|c|c|c|c|c|c|c|c|c|c|c|}
\hline \multirow{2}{*}{ No. of layers } & \multirow{2}{*}{ Theory } & \multicolumn{9}{|c|}{$a / h$} \\
\hline & & 2 & 4 & 5 & 10 & 12.5 & 20 & 25 & 50 & 100 \\
\hline \multirow{3}{*}{$\left(45^{\circ} /-45^{\circ}\right)_{1}$} & Present Model & 6.3247 & 9.7517 & 10.8336 & 13.2605 & 13.7058 & 14.2455 & 14.3823 & 14.5722 & 14.6211 \\
\hline & Reddy [11] & 6.2837 & 9.7593 & 10.8401 & 13.2630 & 13.7040 & 14.2463 & 14.3827 & 14.5723 & 14.6214 \\
\hline & Swaminathan [29] & 5.3325 & 8.8426 & 10.0350 & 12.9115 & 13.4690 & 14.1705 & 14.3500 & 14.6012 & 14.6668 \\
\hline \multirow{3}{*}{$\left(45^{\circ} /-45^{\circ}\right)_{2}$} & Present Model & 6.1019 & 10.6508 & 12.5342 & 18.3240 & 19.7645 & 21.8072 & 22.3804 & 23.2238 & 23.4508 \\
\hline & Reddy [11] & 6.1067 & 10.6507 & 12.5331 & 18.3221 & 19.7621 & 21.8063 & 22.3798 & 23.2236 & 23.4507 \\
\hline & Swaminathan [29] & 5.5674 & 10.0731 & 11.9465 & 17.8773 & 19.4064 & 21.6229 & 22.2554 & 23.1949 & 23.4499 \\
\hline \multirow{3}{*}{$\left(45^{\circ} /-45^{\circ}\right)_{4}$} & Present Model & 6.3049 & 10.9870 & 12.9697 & 19.2659 & 20.8885 & 23.2390 & 23.9092 & 24.9046 & 25.1745 \\
\hline & Reddy [11] & 6.2836 & 10.9905 & 12.9719 & 19.2659 & 20.8884 & 23.2388 & 23.9091 & 24.9046 & 25.1744 \\
\hline & Swaminathan [29] & 5.9234 & 10.7473 & 12.7523 & 19.1258 & 20.7784 & 23.1829 & 23.8713 & 24.8959 & 25.1741 \\
\hline
\end{tabular}

Table 7. The non-dimensional fundamental frequency of the simply supported square plate $(\theta /-\theta / \ldots)\left(E_{1} / E_{2}=40\right)$.

\begin{tabular}{cccccc}
\hline \multirow{2}{*}{ Layers } & Theory & \multicolumn{4}{c}{$a / h$} \\
\cline { 3 - 6 } & & 10 & 20 & 50 & 100 \\
\hline \multirow{3}{*}{$(5 /-5 / 5 /-5 / 5 /-5)$} & Present Model & 15.9840 & 18.0774 & 18.8394 & 18.9568 \\
& Reddy [11] & 14.848 & 17.619 & 18.753 & 18.935 \\
& Song Xiang [31] & 15.405 & 17.943 & 18.942 & 19.206 \\
\hline \multirow{2}{*}{$(30 /-30 / 30 /-30 / 30 /-30)$} & Present Model & 18.3356 & 21.7196 & 23.0815 & 23.2988 \\
& Reddy [11] & 18.170 & 21.648 & 23.067 & 23.295 \\
& Song Xiang [31] & 19.075 & 22.304 & 23.579 & 23.968 \\
\hline \multirow{2}{*}{$(45 /-45 / 45 /-45 / 45 /-45)$} & Present Model & 19.0252 & 22.8770 & 24.4802 & 24.7392 \\
& Reddy [11] & 19.025 & 22.877 & 24.480 & 24.739 \\
& Song Xiang [31] & 20.027 & 23.623 & 25.061 & 25.478 \\
\hline
\end{tabular}

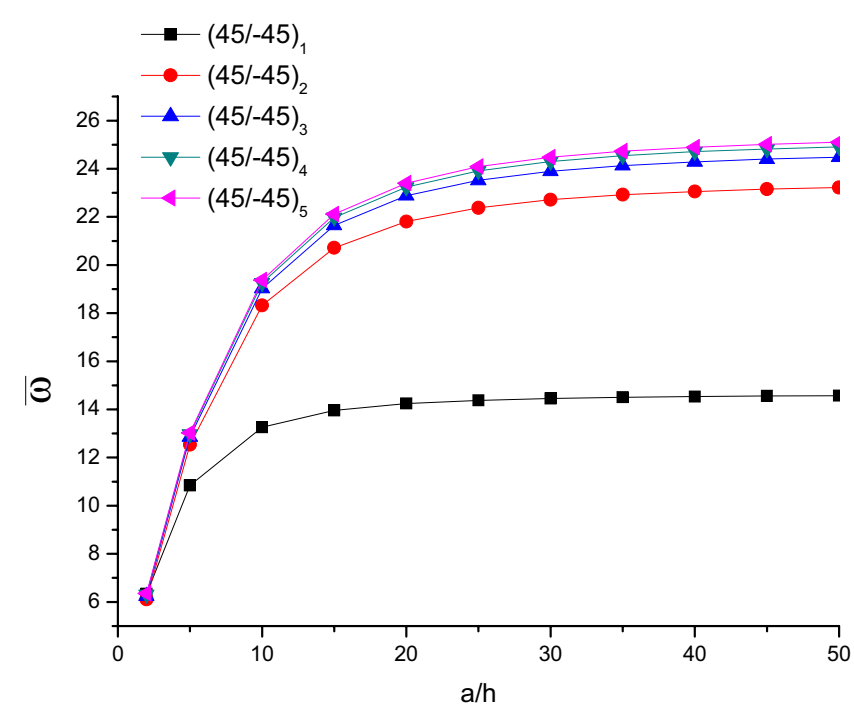

Fig. 5. Variation of dimensionless fundamental frequency of anti-symmetric angle-ply $(45 /-45)_{n}$ square laminates versus thickness ratio (Material 2, $E_{1} / E_{2}=40$ ).

across the plate thickness and satisfies the zero-traction boundary conditions on the top and bottom surfaces of the plate without using shear correction factors. The equations of motion were derived from Hamilton's principle. The accuracy and efficiency of the present model has been demonstrated for buckling and free vibration behav-

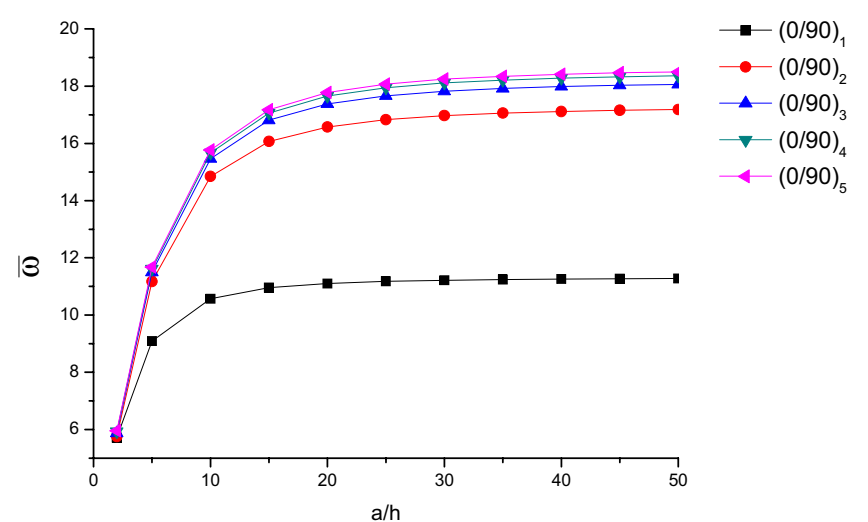

Fig. 6. Variation of dimensionless fundamental frequency of anti-symmetric cross-ply $(0 / 90)_{n}$ square laminates versus thickness ratio (Material 2, $E_{1} / E_{2}=40$ ).

iors of anti-symmetric cross-ply and angle-ply laminates. The conclusions of this theory are as follows:

- The buckling load obtained using the present model with four unknowns and hight order shear deformation Reddy's theory [11] with five unknowns are in good agreement.

- Compared to the three-dimensional elasticity solution, the present model gives more accurate results of buckling load than the height order shear deformation theory. 
B. Adim et al.: Mechanics \& Industry 17, 512 (2016)

Table 8. Dimensionless fundamental frequency of anti-symmetric angle-ply square laminated plate.

\begin{tabular}{|c|c|c|c|c|c|c|c|c|}
\hline \multirow{2}{*}{ No. of layers } & \multirow{2}{*}{$E_{1} / E_{2}$} & \multirow{2}{*}{ Theory } & \multicolumn{6}{|c|}{$a / h$} \\
\hline & & & 2 & 4 & 10 & 20 & 50 & 100 \\
\hline \multirow{20}{*}{$\left(45^{\circ} /-45^{\circ}\right)_{1}$} & \multirow{4}{*}{3} & Present Model & 4.5045 & 6.0858 & 7.0739 & 7.2704 & 7.3293 & 7.3378 \\
\hline & & Reddy [11] & 4.5052 & 6.0861 & 7.0739 & 7.2704 & 7.3292 & 7.3373 \\
\hline & & FSDT [30] & 4.4556 & 6.0665 & 7.0700 & 7.2694 & 7.3291 & 7.3378 \\
\hline & & Swaminathan [29] & 4.5312 & 6.1223 & 7.1056 & 7.3001 & 7.3583 & 7.3666 \\
\hline & \multirow{4}{*}{10} & Present Model & 5.1686 & 7.3454 & 8.9656 & 9.3264 & 9.4377 & 9.4540 \\
\hline & & Reddy [11] & 5.1718 & 7.3469 & 8.9660 & 9.3265 & 9.4377 & 9.4538 \\
\hline & & FSDT $[30]$ & 4.9316 & 7.2169 & 8.9324 & 9.3173 & 9.4362 & 9.4537 \\
\hline & & Swaminathan [29] & 4.9742 & 7.2647 & 8.9893 & 9.3753 & 9.4943 & 9.5123 \\
\hline & \multirow{4}{*}{20} & Present Model & 5.7028 & 8.4115 & 10.7141 & 11.2769 & 11.4553 & 11.4816 \\
\hline & & Reddy [11] & 5.7094 & 8.4151 & 10.7151 & 11.2772 & 11.4553 & 11.4819 \\
\hline & & FSDT $[30]$ & 5.2387 & 8.1185 & 10.6265 & 11.2517 & 11.4511 & 11.4806 \\
\hline & & Swaminathan [29] & 5.1817 & 8.0490 & 10.6412 & 11.2975 & 10.5074 & 11.5385 \\
\hline & \multirow{4}{*}{30} & Present Model & 6.0590 & 9.1696 & 12.0954 & 12.8654 & 13.1153 & 13.1524 \\
\hline & & Reddy [11] & 6.0681 & 9.1752 & 12.0971 & 12.8659 & 13.1154 & 13.1521 \\
\hline & & FSDT [30] & 5.4104 & 8.7213 & 11.9456 & 12.8208 & 13.1077 & 13.1505 \\
\hline & & Swaminathan [29] & 5.2771 & 8.5212 & 11.8926 & 12.8422 & 13.1566 & 13.2035 \\
\hline & \multirow{4}{*}{40} & Present Model & 6.3246 & 9.7517 & 13.2605 & 14.2455 & 14.5722 & 14.6211 \\
\hline & & Reddy [11] & 6.2837 & 9.7593 & 13.2630 & 14.2463 & 14.5723 & 14.6214 \\
\hline & & FSDT $[30]$ & 5.5205 & 9.1609 & 13.0439 & 14.1790 & 14.5608 & 14.6183 \\
\hline & & Swaminathan [29] & 5.3325 & 8.8426 & 12.9115 & 14.1705 & 14.6012 & 14.6668 \\
\hline \multirow{20}{*}{$\left(45^{\circ} /-45^{\circ}\right)_{2}$} & \multirow{4}{*}{3} & Present Model & 4.6545 & 6.4555 & 7.6268 & 7.8650 & 7.9367 & 7.9471 \\
\hline & & Reddy [11] & 4.6546 & 6.4554 & 7.6267 & 7.8649 & 7.9366 & 7.9472 \\
\hline & & FSDT [30] & 4.6519 & 6.4626 & 7.6293 & 7.8657 & 7.9368 & 7.9472 \\
\hline & & Swaminathan [29] & 4.6498 & 6.4597 & 7.6339 & 7.8724 & 7.9442 & 7.9545 \\
\hline & \multirow{4}{*}{10} & Present Model & 5.3882 & 8.5126 & 11.4678 & 12.2381 & 12.4867 & 12.5236 \\
\hline & & Reddy [11] & 5.3887 & 8.5119 & 11.4674 & 12.2380 & 12.4866 & 12.5238 \\
\hline & & FSDT [30] & 5.3765 & 8.5634 & 11.4939 & 12.2463 & 12.4881 & 12.5239 \\
\hline & & Swaminathan [29] & 5.2061 & 8.3447 & 11.4116 & 12.2294 & 12.4952 & 12.5351 \\
\hline & \multirow{4}{*}{20} & Present Model & 5.7414 & 9.6863 & 14.6619 & 16.3150 & 16.8964 & 16.9851 \\
\hline & & Reddy [11] & 5.7431 & 9.6855 & 14.6609 & 16.3146 & 16.8964 & 16.9848 \\
\hline & & FSDT [30] & 5.6542 & 9.7575 & 14.7292 & 16.3394 & 16.9008 & 16.9862 \\
\hline & & Swaminathan [29] & 5.4140 & 9.3306 & 14.4735 & 16.2570 & 16.8949 & 16.9927 \\
\hline & \multirow{4}{*}{30} & Present Model & 5.9449 & 10.2790 & 16.7765 & 19.3506 & 20.3278 & 20.4806 \\
\hline & & Reddy [11] & 5.9481 & 10.2785 & 16.7750 & 19.3499 & 20.3277 & 20.4807 \\
\hline & & FSDT [30] & 5.7641 & 10.3391 & 16.8825 & 19.3944 & 20.3361 & 20.4827 \\
\hline & & Swaminathan [29] & 5.5079 & 9.7966 & 16.4543 & 19.2323 & 20.3134 & 20.4839 \\
\hline & \multirow{4}{*}{40} & Present Model & 6.1019 & 10.6507 & 18.3240 & 21.8072 & 23.2239 & 23.4508 \\
\hline & & Reddy [11] & 6.1067 & 10.6507 & 18.3221 & 21.8063 & 23.2236 & 23.4507 \\
\hline & & FSDT [30] & 5.8228 & 10.6839 & 18.4633 & 21.8722 & 23.2368 & 23.4541 \\
\hline & & Swaminathan [29] & 5.5674 & 10.0731 & 17.8773 & 21.6229 & 23.1949 & 23.4499 \\
\hline
\end{tabular}

- The natural frequencies obtained by the proposed model with four unknowns are almost identical to those predicted by the shear deformation theories containing five unknowns.

It can be concluded that the present model proposed is accurate in solving the buckling behaviors of anti-symmetric cross-ply and angle-ply laminated composite plates and efficient in predicting the vibration responses of composite plates.
Acknowledgements. The authors thank the referees for their valuable comments.

\section{References}

[1] E. Reissner, The effect of transverse shear deformation on the bending of elastic plates, J. Appl. Mech. Trans. ASME 12 (1945) 69-77 
[2] R.D. Mindlin, Influence of rotary inertia and shear on flexural motions of isotropic, elastic plates, J. Appl. Mech. Trans. ASME 18 (1951) 31-8

[3] Y. Stavski, On the theory of symmetrically heterogeneous plates having the same thickness variation of the elastic moduli, Topics in Applied Mechanics. American Elsevier, New York, 1965, p. 105

[4] P.C. Yang, C.H. Norris, Y. Stavsky, Elastic wave propagation in heterogeneous plates, Int. J. Solids Struct. 2 (1966) 665-684

[5] S. Srinivas, A.K. Rao, Bending, vibration and buckling of simply supported thick orthotropic rectangular plates and laminates, Int. J. Solids Struct. 6 (1970) 1463-1481

[6] J.M. Whitney, C.T. Sun, A higher-order theory for extensional motion of laminated composites, J. Sound Vib. 30 (1973) 85-97

[7] C.W. Bert, Structure design and analysis: Part I, in: C. C. Chamis (Ed.), Analysis of Plates. Academic Press, New York (Chap. 4), 1974

[8] L. Librescu, On the theory of anisotropic elastic shells and plates, Int. J. Solids Struct. 3 (1967) 53-68

[9] M. Levinson, An accurate, simple theory of the statics and dynamics of elastic plates, Mech. Res. Commun. 7 (1980) 343-350

[10] A. Bhimaraddi, L.K. Stevens, A higher order theory for free vibration of orthotropic, homogeneous and laminated rectangular plates, J. Appl. Mech. Trans. ASME 51 (1984) 195-198

[11] J.N. Reddy, A simple higher-order theory for laminated composite plates, J. Appl. Mech. Trans. ASME 51 (1984) $745-752$

[12] J.G. Ren, A new theory of laminated plate, Compos. Sci. Technol. 26 (1986) 225-239

[13] T. Kant, B.N. Pandya, A simple finite element formulation of a higher-order theory for unsymmetrically laminated composite plates, Compos. Struct. 9 (1988) 215264

[14] P.R. Mohan, B.P. Naganarayana, G. Prathap, Consistent and variationally correct finite elements for higher-order laminated plate theory, Compos. Struct. 29 (1994) 445456

[15] A.K. Noor, W.S. Burton, Assessment of shear deformation theories for multilayered composite plates, Appl. Mech. Rev. 42 (1989) 1-13

[16] J.N. Reddy, A review of refined theories of laminated composite plates, Shock. Vib. Dig. 22 (1990) 3-17

[17] J.N. Reddy, An evaluation of equivalent-single-layer and layerwise theories of composite laminates, Compos. Struct. 25 (1993) 21-35
[18] M. Mallikarjuna, T. Kant, A critical review and some results of recently developed refined theories of fiber-reinforced laminated composites and sandwiches, Compos. Struct. 23 (1993) 293-312

[19] L. Dahsin, L. Xiaoyu, An overall view of laminate theories based on displacement hypothesis, J. Compos. Mater. 30 (1996) 1539-1561

[20] R.P. Shimpi, Refined plate theory and its variants, AIAA J. 40 (2002) 137-146

[21] R.P. Shimpi, H.G. Patel, A two variable refined plate theory for orthotropic plate analysis, Int. J. Solids Struct. 43 (2006) 6783-6799

[22] R.P. Shimpi, H.G. Patel, Free vibrations of plate using two variable refined plate theory, J. Sound Vib. 296 (2006) 979-999

[23] A.K. Noor, Stability of multilayered composite plate, Fibre Sci. Technol. 8 (1975) 81-89

[24] J.M. Whitney, N.J. Pagano, Shear deformation in heterogeneous anisotropic plates, J. Appl. Mech. Trans. ASME 37 (1970) 1031-1036

[25] J.G. Ren, Bending, vibration and buckling of laminated plates, in: N.P. Cheremisinoff (Ed.), Handbook of ceramics and composites, Marcel Dekker, New York, 1990, Vol. 1, pp. $413-50$

[26] K. Noor, Free vibrations of multilayered composite plates, AIAA J. 11 (1973) 1038-1039

[27] J.M. Whitney, N.J. Pagano, Shear deformation in heterogeneous anisotropic plates, J. Appl. Mech. 37 (1970) $1031-1036$

[28] M. Karama, K.S. Afaq, S. Mistou, Mechanical behavior of laminated composite beam by the new multi-layered laminated composite structures model with transverse shear stress continuity, Int. J. Solids Struct. 40 (2003) 15251546

[29] K. Swaminathan, S. Patil, Analytical solutions using a higher order refined computational model with 12 degrees of freedom for the free vibration analysis of antisymmetric angle-ply plates, Compos. Struct. 82 (2008) 209-216

[30] Huu-Tai Thai, Dong-Ho Choi, A simple first-order shear deformation theory for laminated composite plates, Compos. Struct. 106 (2013) 754-763

[31] Song Xiang, Guang-chao Li, Wei Zhang, Ming-sui Yang A meshless local radial point collocation method for free vibration analysis of laminated composite plates, Compos. Struct. 93 (2011) 280-286

[32] K. Nedri, N. El Meiche, A. Tounsi, Free vibration analysis of laminated composite plates resting on elastic foundations by using a refined hyperbolic shear deformation theory, J. Mech. Comp. Mat. 49 (2013) 943-958 